\title{
Using hydrogel-biochar composites for enhanced cadmium removal from aqueous media
}

\begin{abstract}
Cadmium $(\mathrm{Cd})$ is a common heavy metal and is often released into aquatic environment during mining activities and metallurgical processes. At high concentrations, $\mathrm{Cd}$ is highly toxic to most organisms and can be accumulated in organisms before entering human body through food chains. Many technologies have been developed to remediate Cd contamination, among which biochar is one of the most sustainable and promising. However, high pricing of biochar today is plaguing its large-scale applications. Therefore, in this study, inexpensive acrylamide was used to synthesize polyacrylamide hydrogel-biochar composites with the dosages of 1,3 and $5 \%$ weight ratios of biochar. The swelling ratio significantly increased from $818 \%$ for untreated hydrogel to 1220,1349 and $1506 \%$ for composites with increasing dosages of biochar, respectively. According to the isotherm experiment results, modeling simulations indicated that the maximum adsorption capacities (qmax) of the composites were $30.63,59.21$ and $63.58 \mathrm{mg} / \mathrm{g}$ for the three composites with increasing biochar dosages, respectively. In comparison, the untreated hydrogel had a much lower qmax of 24.72 $\mathrm{mg} / \mathrm{g}$. Besides the greater water swelling capacity, the significantly improved ability of hydrogel-biochar composites to entrap $\mathrm{Cd}$ from aqueous media was hypothetically attributed to the formation of ionic attraction fields around scattered biochar particles. It was recommended that $3 \%$ of biochar be the optimal dosage because further improvement became insignificant at higher dosages. The use of hydrogel-biochar composites for $\mathrm{Cd}$ contamination issues is a cost-effective approach.
\end{abstract}

Keywords: heavy metal; cadmium; hydrogel; biochar; hydrogel-biochar composite; swelling capacity; sorption isotherm.
Special Issue - 2018

Simeng Li, Gang Chen

Department of Civil and Environmental Engineering, USA

Correspondence: Simeng Li, Department of Civil and Environmental Engineering, USA, Tel + I 850645 0I26, EmailsII6b@my.fsu.edu

Received: September 28, 2018 | Published: December 31, 2018

\section{Introduction}

Mining activities and metallurgical processes often introduce considerable amounts of heavy metals to the surrounding environment; especially the nearby water bodies as well as groundwater system. ${ }^{1}$ High concentrations of most heavy metals are highly toxic to living organisms including humans. Moreover, many heavy metals can accumulate in organisms (i.e., bioaccumulation) and be consumed by other organisms along the food chain. ${ }^{2}$ among the common heavy metals, cadmium $(\mathrm{Cd})$, which is mostly sourced from zinc refinery, has the highest toxicity to most organisms. ${ }^{3}$ Inhalation of high levels of $\mathrm{Cd}$ in a short period of time can lead to acute flu-like symptoms and lung damages, while long-term exposure to $\mathrm{Cd}$ can result in serious kidney, bone and lung diseases. ${ }^{4}$ In addition, cadmium and its compounds are also known to be carcinogenic. ${ }^{5}$ Therefore, removing heavy metals, particularly $\mathrm{Cd}$ from the contaminated aquatic environment has long been an important challenge.

Many sustainable technologies have been developed for heavy metal removal in the past decade, among which biochar seems to be one of the most promising. ${ }^{6-8}$ Biochar is the solid product of biomass pyrolysis, an oxygen-limiting/-absent high-temperature heating process for biofuel production. ${ }^{9}$ Considering its significant adsorptive ability due to its high specific surface area and diverse surface functional groups, biochar has been often used as an adsorbent for soil and water remediation. ${ }^{7}$ For example, with $3 \%$ of biochar application in agricultural soil, Abbas et al. reported that $\mathrm{Cd}$ concentration in wheat grains decreased by $42 \% .{ }^{10}$ The maximum adsorption capacities of sesame straw biochar (produced at $700^{\circ} \mathrm{C}$ for $4 \mathrm{~h}$, sieved to $<0.5 \mathrm{~mm}$ ) for $\mathrm{Cd}$ in aqueous solution was reported to be as high as $86 \mathrm{mg} / \mathrm{g} .{ }^{11}$ However, the high pricing of biochar in the market is currently plaguing its large-scale applications. ${ }^{9}$ Therefore, the development of more economic biochar production processes or alternative technologies is in an urgent need in order to address the ecological problems caused by heavy metals in the environment.

A hydrogel is superabsorbent that can soak up liquid easily and store over 1000 times of its original weight of liquid inside its crosslinked polymeric structure. ${ }^{12}$ Therefore, the use of hydrogels in agriculture as soil water reservoirs is prevalent ${ }^{12}$. In addition, hydrogel production is rapid and inexpensive. The most common monomer for polymerizing hydrogel is acrylamide, whose market price can be as low as $\$ 1$ per kilogram. However, the ability of polyacrylamide hydrogel to entrap heavy metals is much less significant than that of biochar. Hence, in order to improve the effectiveness of polyacrylamide hydrogel, many efforts have been made. ${ }^{13,14}$

In this study, polyacrylamide hydrogel composites with different weight ratios (i.e., $1 \%, 3 \%$ and $5 \%$ ) of biochar were synthesized for the removal of $\mathrm{Cd}$ from aqueous solutions. The influence of biochar dosage was compared and discussed. It was conjectured that hydrogel composites with small amount of incorporated biochar could effectively enhance the entrapment of $\mathrm{Cd} 2+$ ions, thus increasing the removal of $\mathrm{Cd}$. The use of hydrogel-biochar composite can be a costeffective solution for heavy metal contamination issues. 


\section{Materials and methods}

\section{Materials}

The hydrogels used in this work were crosslinked polyacrylamide polymers (Soil Moist $\AA$ containing $99.7 \%$ crosslinked polyacrylamide and $0.3 \%$ inert ingredients) provided by JRM Chemical Inc. (Cleveland, $\mathrm{OH}$ ) as a gift. The tested biochar was Black Owl Biochar ${ }^{\circledR}$ (Environmental Ultra TM) purchased from Biochar Supreme, LLC (Everson, WA). According to the manufacturer, the biochar was pyrolyzed from wood wastes and had high organic carbon content greater than $80 \%$. The hydrogel-biochar composites were synthesized in the laboratory using acrylamide as monomer, N,N'methylenebisacrylamide as crosslinker, and ammonium persulfate as initiator. All the materials were stored in a desiccator before being used.

\section{Preparation of hydrogel-biochar composites}

The hydrogel-biochar composites were prepared at three different biochar-to-composite weight ratios, i.e., $1 \%, 3 \%$ and $5 \%$. Prior to the polymerization, the biochar was washed with $0.1 \mathrm{mM} \mathrm{HCl}$ for $6 \mathrm{~h}$ followed by deionized (DI) water until its $\mathrm{pH}$ was neutral, and then dried in an oven at $650 \mathrm{C}$ for 72 hours. The purpose of this pretreatment was to remove the minerals on the biochar surface, i.e., activation. ${ }^{15}$ the synthesis of hydrogel-biochar composite was conducted using acrylamide and pretreated biochar as the building units. In brief, $0.792 \mathrm{~g}$ of acrylamide was weighed and dissolved in $1.0 \mathrm{~mL}$ of water. Subsequently, the pretreated biochar $(0.1 \mathrm{~g}$ for $1 \%, 0.3 \mathrm{~g}$ for $3 \%$ and $0.5 \mathrm{~g}$ for $5 \%$ ), together with $0.108 \mathrm{~g}$ of $\mathrm{N}, \mathrm{N}$ '-methylenebisacrylamide, was added to the acrylamide solution. The mixture was thoroughly mixed using a vortex mixer (VWR, Radnor, PA). Afterwards, $0.2 \mathrm{~mL}$ of $0.07 \mathrm{mM}$ ammonium persulfate solution was used to initiate the polymerization and crosslinking. The hydrogel precursor solution was stored in 3-mm (inner diameter) cylindrical pipettes at room temperature for $12 \mathrm{~h}$. The resulting solid hydrogels were cut into $3 \mathrm{~mm}$ in length. The unreacted monomers as well as small-molecular-weight polymeric matter were then washed off with distilled water. The final composites were dried in air at room temperature for $24 \mathrm{~h}$ and then in a vacuum oven at $40^{\circ} \mathrm{C}$ for $48 \mathrm{~h}$.

\section{Swelling ratio}

The swelling ratio was used to characterize the hydrogels and hydrogel-biochar composites. Approximately $0.05 \mathrm{~g}$ of hydrogel or hydrogel-biochar composite was placed in DI water. The weight increase was monitored at room temperature by weighing the material that was periodically removed from the DI water. Each measurement was repeated for at least three times. The swelling ratio was calculated using the following equation (Eq. (1)).

\section{Swelling ratio $(\%)=\left(\mathrm{W} \_\right.$swollen-W_dry $) / \mathrm{W}$ dry $\times 100$ (1)}

Where, Wswollen is the measured weight of material after contact with DI water, and Wdry is the measured weight of dry material.

\section{Measurement of cadmium in aqueous media}

Stock Cd solution of $1000 \mathrm{mg} / \mathrm{L}$ were prepared by dissolving the exact quantity of $\mathrm{Cd}(\mathrm{NO} 3) 2 \cdot 4 \mathrm{H} 2 \mathrm{O}$ in DI water. The concentrations of $\mathrm{Cd}$ in this study were determined using a Microwave Plasma-Atomic Emission Spectrometer (4200 MP-AES, Agilent, Santa Clara, CA).

\section{Sorption experiments}

Following the procedure described in a previous study, ${ }^{16}$ batch sorption experiments were conducted at room temperature to investigate the sorption of cadmium by the hydrogel, biochar, and hydrogel-biochar composite. A series of solutions $(20 \mathrm{~mL})$ with cadmium concentrations of $50,100,200,400$, and $800 \mathrm{mg} / \mathrm{L}$ were added into $25-\mathrm{mL}$ polyethylene centrifuge tubes, together with $0.05 \mathrm{~g}$ of the sorbent. The mixture was agitated on a wrist-action shaker (Model 75 , Burrel Scientific, Pittsburg, PA) for $72 \mathrm{~h}$ to reach equilibrium. The amount of $\mathrm{Cd}$ adsorbed by the sorbent was calculated as below (Eq. (2)).

Where, $\mathrm{q}(\mathrm{mg} / \mathrm{g})$ is the amount of adsorbed Cd per unit mass of sorbent, $\mathrm{C} 0(\mathrm{mg} / \mathrm{L})$ and $\mathrm{Ce}(\mathrm{mg} / \mathrm{L})$ are respectively the initial and equilibrium concentration of $\mathrm{Cd}, \mathrm{V}(\mathrm{L})$ is the volume of $\mathrm{Cd}$ solution added for the sorption, and $\mathrm{M}(\mathrm{g})$ is the mass of sorbent added for the sorption.

To estimate the maximum adsorption capacity of the sorbent, the data obtained from sorption experiments were best-fitted and simulated using the Langmuir sorption isotherm model. ${ }^{17}$

\section{Statistical analysis}

The means and errors of the experimental data were statistically analyzed with the use of SPSS for Windows 14.0 (IBM, Armonk, $\mathrm{NY}$ ). Based on the analysis of variance (ANOVA) performed with GraphPad ${ }^{\circledR}$ Prism, a significance level of $5 \%(\mathrm{p}<0.05)$ was used to evaluate the differences between different tested values. ${ }^{18}$

\section{Results and discussion}

\section{Swelling capacity of hydrogels and composites}

The swelling ratio is one of the most important parameters for evaluating the effectiveness of hydrogel materials. After $48 \mathrm{~h}$ of swelling in DI water, both the polyacrylamide hydrogel and hydrogelbiochar composites reached their maximum swelling capacities (Figure 1). It was observed that the hydrogel-biochar composites were able to swell too much larger amounts of water comparing to the untreated polyacrylamide hydrogel. The swelling capacity was found to increase more with a higher weight ratio of biochar in the composites, which was probably due to the presence of hydrophilic functional groups introduced by biochar. ${ }^{9}$ The swelling ratios of the tested hydrogel materials were in the order (from large to small): hydrogel composite with $5 \%$ biochar $>$ hydrogel composite with $3 \%$ biochar $>$ hydrogel composite with $1 \%$ biochar $>$ untreated hydrogel. The results showed consistency with the levels of hydrophilicity of the hydrogel materials. The experimental results were also bestfitted with hyperbolic nonlinear regressions $(p<0.001)$ to estimate the maximum swelling capacities. The fitting results indicated that the maximum swelling ratios were $818 \%$ for untreated hydrogel, $1220 \%$ for hydrogel composite with $1 \%$ biochar, $1349 \%$ for hydrogel composite with $3 \%$ biochar, and $1506 \%$ for hydrogel composite with $5 \%$ biochar. The above results showed that the water swelling capacities of the hydrogel-biochar composites was significantly better than that of the untreated polyacrylamide hydrogel, implying that the composites might be more appropriate for environmental applications in aqueous media. 


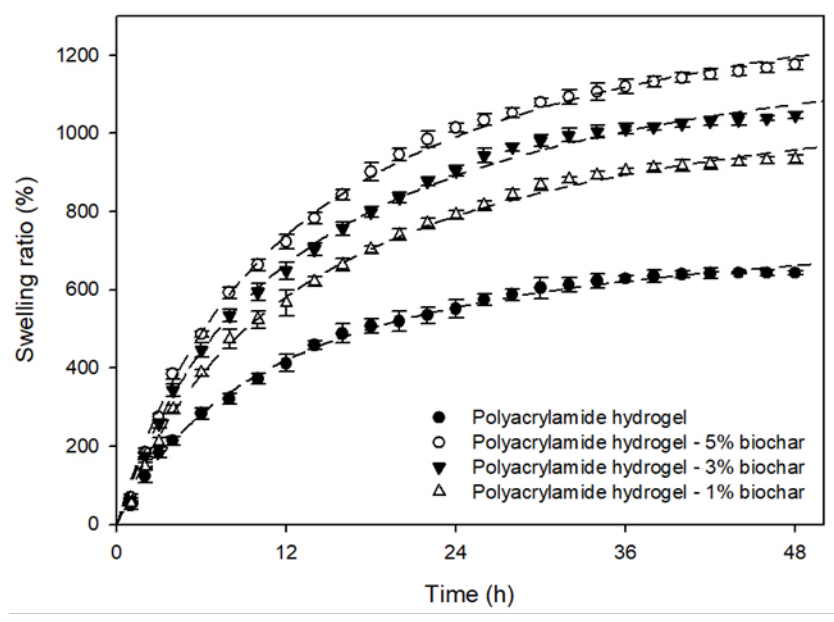

Figure I Swelling of hydrogel material in DI water as a function of time.

\section{Sorption of cadmium by biochar}

Biochar demonstrated effective removal of $\mathrm{Cd}$ from the aqueous media (Figure 2). The equilibrium adsorption capacity (qe), i.e., the adsorbed mass of $\mathrm{Cd}$ per unit weight of biochar at equilibrium, consistently increased with the increasing initial $\mathrm{Cd}$ concentration (Figure 2a). This increasing trend became less pronounced at higher concentrations because the sorbent (i.e., biochar) was approaching its maximum adsorption capacity (qmax). In Figure 2b, qe was plotted against the concentrations of $\mathrm{Cd}$ at equilibrium $(\mathrm{Ce})$. By simulating the experimental data using Langmuir isotherm model, the isotherm curve (dash line) of $\mathrm{Cd}$ sorption by biochar was generated (R2 = 0.954). The maximum adsorption capacity (qmax) of the tested biochar for $\mathrm{Cd} 2+$ was estimated to be $88.03 \mathrm{mg} / \mathrm{g}(\mathrm{p}$-value $=0.004)$, which was similar to the results reported in previous studies. ${ }^{8,11,15}$ The results above showed that the biochar used for producing the hydrogelbiochar composites in this study had a good ability for attracting and retaining $\mathrm{Cd} 2+$ ions. The maximum adsorption capacity of biochar $(88.03 \mathrm{mg} / \mathrm{g})$ was also used as a reference for that of the hydrogel and its composites.

\section{Sorption of cadmium by hydrogel materials}

Similar to the increasing trend of biochar, the sorption of $\mathrm{Cd}$ by polyacrylamide hydrogel and its composites also increased as the initial concentration of $\mathrm{Cd}$ increased (Figure 3a). It was obvious that the removal of $\mathrm{Cd}$ was significantly enhanced with the small amount of incorporated biochar in the hydrogel-biochar composites. The degree of improvement was greater when a higher weight ratio of biochar was incorporated. According to the sorption isotherms of $\mathrm{Cd}$ and simulations of the experimental results using Langmuir isotherm model (Figure 3b), R2=0.954-0.996), the maximum adsorption capacity of the untreated polyacrylamide hydrogel was $24.73 \mathrm{mg} / \mathrm{g}$ (p-value $=0.027$ ), of the composite with $1 \%$ biochar was $30.63 \mathrm{mg} / \mathrm{g}$ $(\mathrm{p}$-value $=0.001)$, of the composite with $3 \%$ biochar was $59.21 \mathrm{mg} / \mathrm{g}$ (p-value $=0.001)$, and of the composite with $5 \%$ biochar was 63.58 $\mathrm{mg} / \mathrm{g}(\mathrm{p}$-value $=0.001)$, as shown in Table 1 . These results showed that the sorption of $\mathrm{Cd}$ by the hydrogel composites were greatly improved due to the presence of biochar within the crosslinked structures. Besides the increased swelling capacities of these composites, the improvement can also be attributed to the ionic and magnetic attractions introduced by the diverse functional groups on the surface of biochar. These surface functional groups, which have been identified in our previous studies, ${ }^{9,17}$ include carboxylic acid, amine, hydroxyl and sulfonic acid groups, etc.

Interestingly, the increased maximum adsorption capacities (qmax) of the hydrogel composites were significantly larger than solely the sums of the qmax of the untreated polyacrylamide hydrogel and the qmax of the incorporated biochar for the synthesis. The results demonstrated that the combination of polyacrylamide hydrogel and biochar was synergistic in terms of the composites' capacities of removing $\mathrm{Cd}$ from aqueous media. The reason for this synergistic effect was probably that each single biochar particle scattered in the composite created an ionic attraction field for attracting and retaining metallic ions such as $\mathrm{Cd} 2+$. This ionic attraction field significantly extended the potential of each biochar particle to capture ions. Within the cross linked structures of the hydrogel-biochar composites, high concentrations of metallic ions could be entrapped against a larger concentration gradient of ions. The formation of many ionic attraction fields around the scattered biochar particles made it possible to overcome the ionic osmotic stress, the main factor regulating the sorption and release of ions in hydrogel materials. ${ }^{19}$
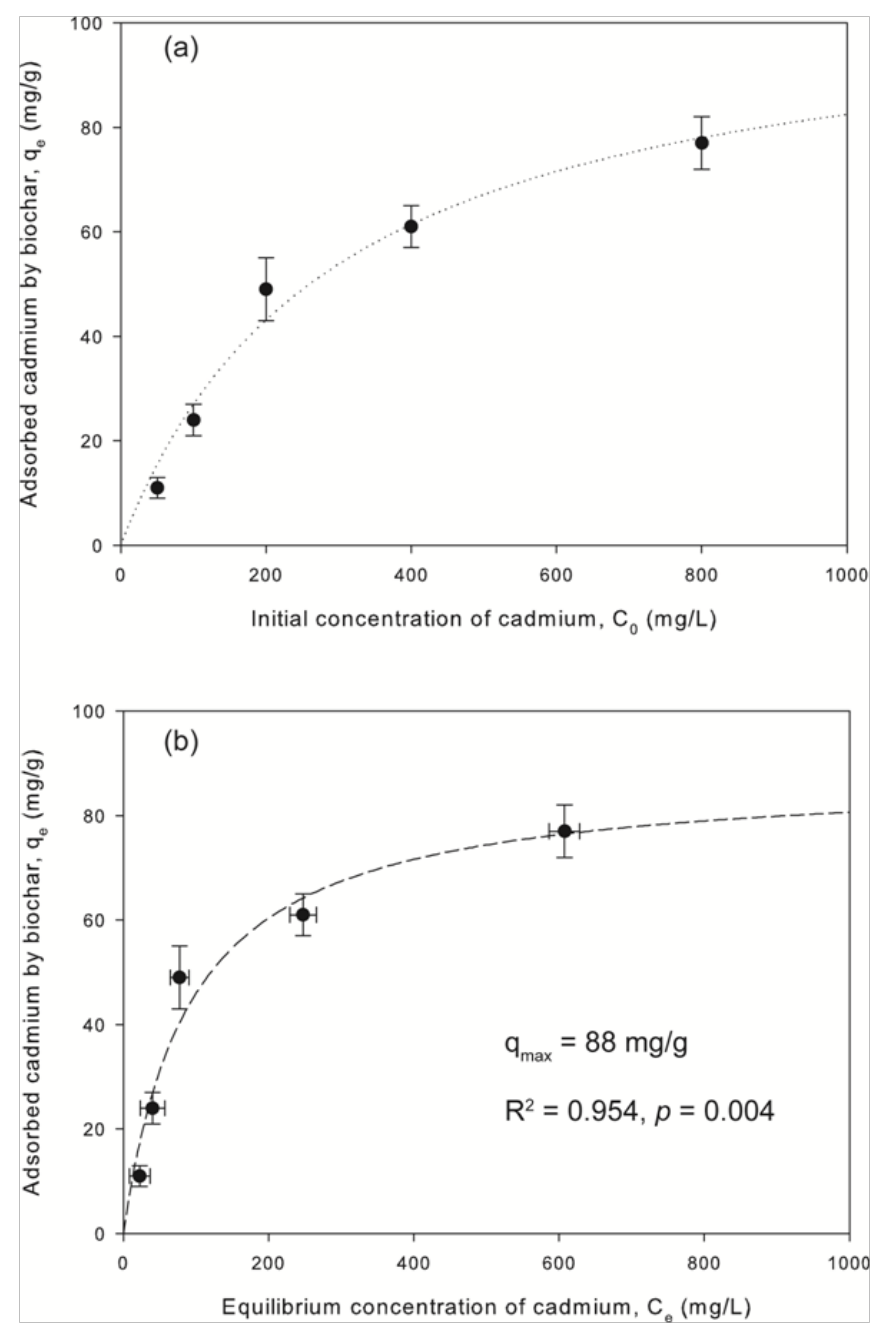

Figure 2 Sorption of cadmium by biochar: (a) influence of initial cadmium concentration; (b) isotherm curve of cadmium sorption simulated by Langmuir model. 
Table I Summary of sorption kinetics of cadmium adsorption by biochar and hydrogel materials

\begin{tabular}{llll}
\hline Absorbent & qmax $(\mathbf{m g} / \mathbf{g})$ & $\mathbf{R}^{2}$ & p-value \\
\hline Biochar & 88.03 & 0.954 & 0.004 \\
Hydrogel & 24.72 & 0.973 & 0.027 \\
Composite with 1\% biochar & 30.63 & 0.994 & 0.001 \\
Composite with 3\% biochar & 59.21 & 0.996 & 0.001 \\
Composite with 5\% biochar & 63.58 & 0.995 & 0.001 \\
\hline
\end{tabular}
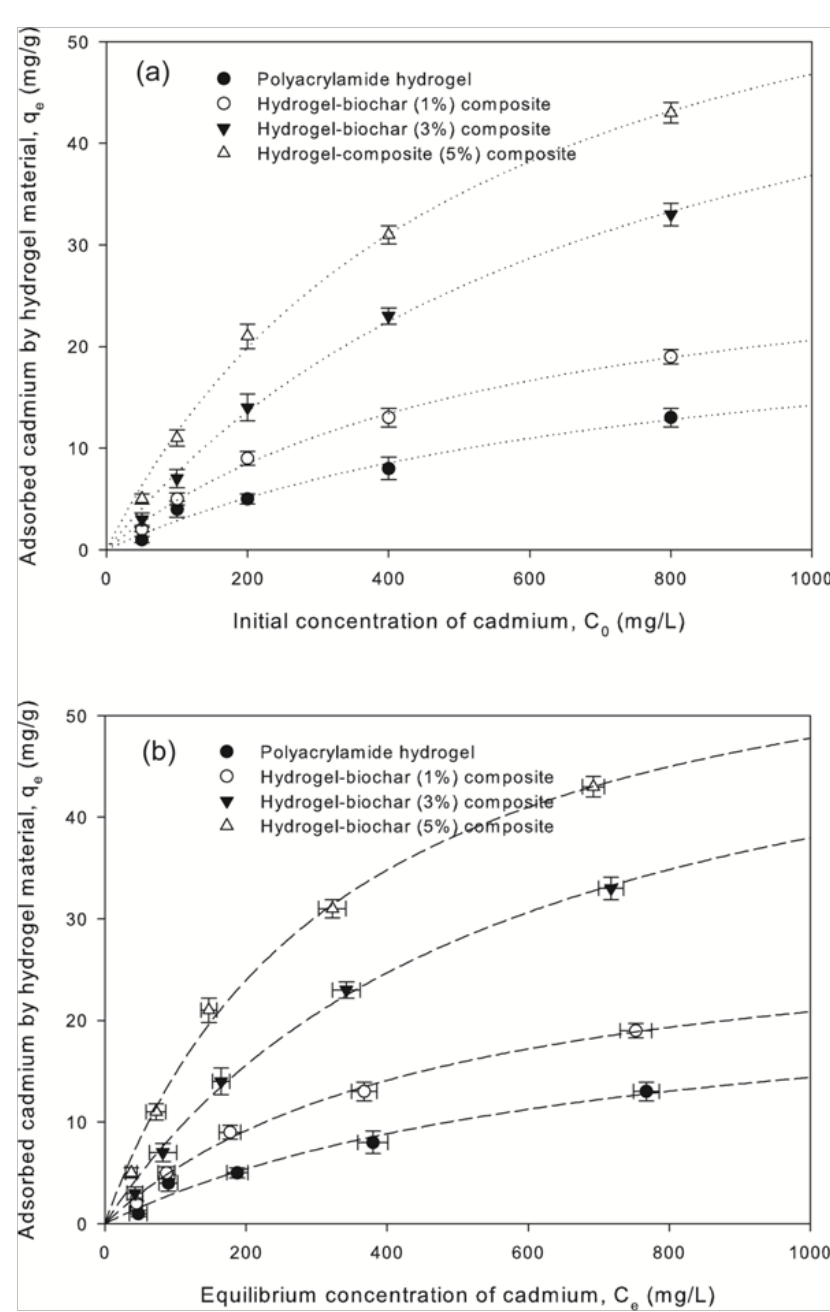

Figure 3 Sorption of cadmium by hydrogel material: (a) influence of initial cadmium concentration; (b) isotherm curve of cadmium sorption simulated by Langmuir model.

\section{Influence of biochar dosage for hydrogel-biochar composite}

The usage of synthesized hydrogel-biochar composites with biochar weight ratios of 1,3 and $5 \%$ increased the Cd sorption by $23.9 \%, 139.5 \%$ and $157.2 \%$, respectively (Figure 4). Notably, the fitting curve (dash line) generated from linear regression derived an unsatisfactory correlation $(\mathrm{R} 2=0.848)$, suggesting that the synergistic effect of hydrogel-biochar composite does not linearly increase with the dosage of biochar used for the syntheses. Instead, the experimental data fitted well with sigmoid nonlinear regression (dotted line). When a small dosage of biochar (e.g., $<1 \%$ ) was used for the composite synthesis, the increase of $\mathrm{Cd}$ sorption $(<20 \%)$ was obvious although less sound comparing to the increase (30-150\%) when a relatively higher dosage of biochar (e.g., 1-3\%) was used. However, when more biochar ( $>3 \%$ ) was used for the synthesis of hydrogel-biochar composite, the improvement of $\mathrm{Cd}$ sorption became much less significant. These results complied with the hypothesis that an ionic attraction field was formed around each biochar particle. With the increasing number of scattered biochar particles, the average distance between each particle became smaller, thus creating more mutual coverages of different ionic attraction fields by different biochar particles. When the crosslinked space of the composite became more crowded and saturated, the overall effectiveness of the ionic attraction fields diminished as a result. These results also indicated that there would be no need to level up the dosage of biochar to more than $3 \%$ when synthesizing polyacrylamide hydrogel-biochar composites for Cd removal. A higher dosage of biochar $(>3 \%)$ is not only economically inefficient; it also does not bring significant further improvement.

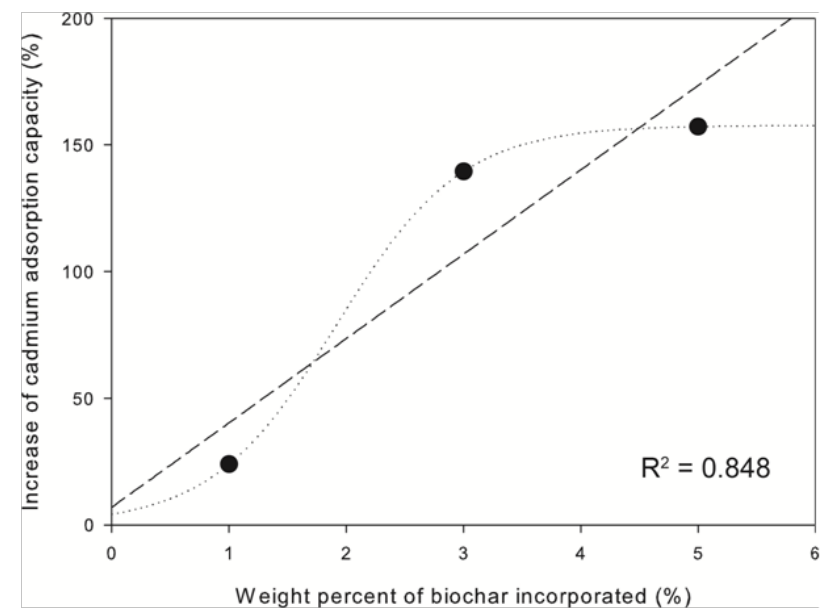

Figure 4 Increase of cadmium adsorption capacity as a function of the amount of incorporated biochar in hydrogel-biochar composite.

\section{Conclusion}

In order to improve the effectiveness of polyacrylamide hydrogel in removing $\mathrm{Cd}$ from aqueous media, small amounts of biochar at three different weight ratios (i.e., 1,3 and $5 \%$ ), together with acrylamide as monomer, were used for synthesizing hydrogel-biochar composites. The resulting composites had significantly improved ability to entrap water and $\mathrm{Cd} 2+$ ions, and therefore was more capable of removing these ions from aqueous media. The swelling ratio of the hydrogelbiochar composite with $5 \%$ biochar was almost doubled comparing to the untreated hydrogel. With significant increases as high as $157.2 \%$, the composites also demonstrated a great potential for $\mathrm{Cd}$ removal from aqueous media. However, a dosage higher than 3\% did not result in significant further improvement for Cd removal. Therefore, the weight ratio of 3\% can be considered optimal for the composite synthesis. Considering the high costs of large-scale biochar application, using hydrogel-biochar composites is a more cost-effective approach for $\mathrm{Cd}$ removal from the contaminated environment. 


\section{Acknowledgement}

Authors acknowledge JMR Chemical Inc. (Cleveland, OH) for sending the Soil Moist ${ }^{\mathbb{B}}$ hydrogel product as a gift to support our research.

\section{Conflict of interests}

Authors declare that there is no conflict of interest.

\section{References}

1. Pour ZS, Ghaemy M. Removal of dyes and heavy metal ions from water by magnetic hydrogel beads based on poly(vinyl alcohol)/carboxymethyl starch-g-poly(vinyl imidazole). Rsc Adv. 2015;5(79):64106-64118.

2. Ip CC, Zhang $\mathrm{G}$, Wong $\mathrm{CSC}$, et al. Heavy metal and $\mathrm{Pb}$ isotopic compositions of aquatic organisms in the Pearl River Estuary, South China. Environ Pollut. 2005;138(3):494-504.

3. Di Lorenzo M, Thomson AR, Schneider K, et al. A small-scale air-cathode microbial fuel cell for on-line monitoring of water quality. Biosens Bioelectron. 2014;62:182-188.

4. Habineza A, Zhai J, Ntakirutimana T, et al. Heavy metal removal from wastewaters by agricultural waste low-cost adsorbents: hindrances of adsorption technology to the large scale industrial application-a review. Desalin Water Treat. 2017;78:192-214.

5. Chen PZ, Duan XH, Li M, et al. Wang, H., Systematic network assessment of the carcinogenic activities of cadmium. Toxicol Appl Pharm. 2016;310:150-158.

6. Ho SH, Zhu SS, Chang JS. Recent advances in nanoscale-metal assisted biochar derived from waste biomass used for heavy metals removal. Bioresource Technol. 2017;246:123-134.

7. Wang B, Gao B, Fang J. Recent advances in engineered biochar productions and applications. Crit Rev Env Sci Tec. 2017;47(22):2158-2207.

8. Komkiene J, Baltrenaite E. Biochar as adsorbent for removal of heavy metal ions [Cadmium(II), Copper(II), Lead(II), Zinc(II)] from aqueous phase. Int J Environ Sci Te. 2016;13(2):471-482.
9. Li S, Chen G. Thermogravimetric, thermochemical, and infrared spectral characterization of feedstocks and biochar derived at different pyrolysis temperatures. Waste Manage. 2018;78:198-207.

10. Abbas T, Rizwan M, Ali Set al. Effect of biochar on cadmium bioavailability and uptake in wheat (Triticum aestivum L.) grown in a soil with aged contamination. Ecotox Environ Safe. 2017;140:37-47.

11. Park JH, Ok YS, Kim SH, et al. Competitive adsorption of heavy metals onto sesame straw biochar in aqueous solutions. Chemosphere. 2016;142:77-83.

12. Guilherme MR, Aouada FA, Fajardo AR, et al. Superabsorbent hydrogels based on polysaccharides for application in agriculture as soil conditioner and nutrient carrier: A review. Eur Polym J. 2015;72:365-385.

13. Zamarripa-Ceron JL, Garcia-Cruz JC, Martinez-Arellano AC, et al. Heavy metal removal using hydroxypropyl cellulose and polyacrylamide gels, kinetical study. J Appl Polym Sci. 2016;133(15).

14. Wang JJ, Li XN. Synthesis of polyacrylamide/modified silica composite hydrogels for synergistic complexation of heavy metal ions. Desalin Water Treat. 2015;3(1):230-237.

15. Abdelhadi SO, Dosoretz CG, Rytwo G, et al. Production of biochar from olive mill solid waste for heavy metal removal. Bioresource Technol. 2017;244:759-767.

16. Yuan, H.; Li, S.; Liu, J.; Song, C.; Chen, G., Cry1Ab adsorption and transport in humic acid-coated geological formation of alumino-silica clays. Water Air Soil Poll. 2017;228:387-394.

17. Li S, Barreto V, Li R, et al. Nitrogen retention of biochar derived from different feedstocks at variable pyrolysis temperatures. J Anal Appl Pyrol. 2018;133:136-146.

18. Li S, Chen G. Factors affecting the effectiveness of bioelectrochemical system applications: Data synthesis and meta-analysis. Batteries. 2018;4:34-51.

19. Wang HT, Wei J, Simon GP. Response to Osmotic Pressure versus Swelling Pressure: Comment on "Bifunctional Polymer Hydrogel Layers As Forward Osmosis Draw Agents for Continuous Production of Fresh Water Using Solar Energy”. Environ Sci Technol. 2014;48(7):4214-4215. 